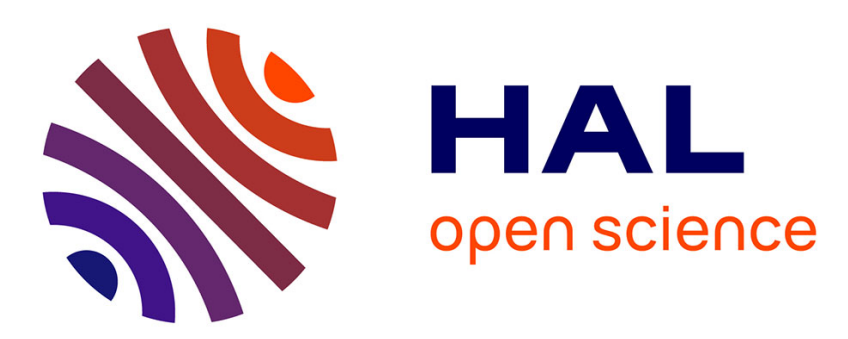

\title{
Polarization sensitive sub-wavelength metallic structures: toward near-field light confinement control
}

\author{
A. Ndao, Q. Vagne, J. Salvi, F.I. Baida
}

\section{To cite this version:}

A. Ndao, Q. Vagne, J. Salvi, F.I. Baida. Polarization sensitive sub-wavelength metallic structures: toward near-field light confinement control. Applied Physics B - Laser and Optics, 2011, 106 (4), pp.857-862. 10.1007/s00340-011-4835-2 . hal-00673403

\section{HAL Id: hal-00673403 \\ https://hal.science/hal-00673403}

Submitted on 13 May 2021

HAL is a multi-disciplinary open access archive for the deposit and dissemination of scientific research documents, whether they are published or not. The documents may come from teaching and research institutions in France or abroad, or from public or private research centers.
L'archive ouverte pluridisciplinaire HAL, est destinée au dépôt et à la diffusion de documents scientifiques de niveau recherche, publiés ou non, émanant des établissements d'enseignement et de recherche français ou étrangers, des laboratoires publics ou privés. 


\title{
Polarization sensitive sub-wavelength metallic structures: toward near-field light confinement control
}

\author{
A. Ndao $\cdot$ Q. Vagne $\cdot$ J. Salvi $\cdot$ F.I. Baida
}

\begin{abstract}
In this paper, we seek to demonstrate spatial near field light confinement with a truly sub-wavelength resolution in the visible range. For that purpose, an opaque metallic screen is pierced with an array of rectangular nano-apertures which support polarization sensitive guided modes. We show that it is possible to switch on and off sub-wavelength apertures about only 200 nanometers away. Theoretical results have been performed (3D-FDTD homemade code) and are in good agreement with the experimental results. These kinds of nano-structures might offer a convenient and versatile way to sub-wavelength light addressing, optical trapping, molecular, or nano-probing, nonlinear spectroscopy.
\end{abstract}

\section{Introduction}

The control of sub-wavelength light confinement is still a big challenge for several applications covering areas ranging from the miniaturization of optical devices to nano-medicine through data storage, single molecule detection, and nanoparticles trapping. The easiest way to meet this constraint of electro-magnetic localization has been, for several years, in the use of optical near field microscope probes [1,2] or nano-antennas [3-5]. Research in this area is still very long focused on the optimization of these probes to allow efficient detection and/or emission of the light field while keeping this character of the local measure. A lot of studies were performed in this area. Leading research groups in

A. Ndao $\cdot$ Q. Vagne $\cdot$ J. Salvi $(\bowtie) \cdot$ F.I. Baida

Département d'Optique P.M. Duffieux, Institut FEMTO-ST UMR

6174 CNRS, Université de Franche-Comté, 25030 Besançon

Cedex, France

e-mail: jerome.salvi@univ-fcomte.fr

Fax: +33-381666423 the world proposed to investigate the tremendous potential of meta-materials [6-9] but the most common issue to this problem consists in making the most of excitation of plasmon resonances on a metallic nano-structure [10-16]. Indeed, surface plasmons offer the possibility to overcome the diffraction limit and plasmonic has opened a wide route to sub-wavelength photonic circuitry [17, 18].

Our purpose here is to demonstrate possible sub- $\lambda$ light localization without plasmon excitation. Guided modes inside apertures engraved into an opaque metallic screen are responsible for the near-field light confinement. This idea was recently proposed by Baida [19], where spatiotemporal control of light confinement is achieved in linear regime by formatting the incident beam simultaneously through its instantaneous polarization, amplitude, and phase. A negative chirped pulse (the frequency decreases versus time) with a specific amplitude modulation allows to light successively the five letters of the nano word "FEMTO"-with the same intensity-in temporal sequences separated by almost 30 fs. The triple control of the incident beam remains a very difficult experimental task that we are presently unable to achieve. Consequently, only spatial control will be addressed in this paper thanks to rectangular nano-slits.

\section{Design of the sub-wavelength metallic structures}

The confinement is obtained by the excitation of the fundamental guided mode of each rectangular nano-aperture. Contrary to the case of a perfect electric conductor for which the cutoff wavelength of the fundamental guided mode of a rectangular waveguide $\lambda_{c}^{\text {fund }}$ is twice the length $a$ of the aperture, $\lambda_{c}^{\text {fund }}$ does not have an analytical expression for a real metal (with dispersion). Nevertheless, and because of the electro-magnetic field penetration into the real metal, the 
Fig. 1 SEM image of the whole fabricated matrix. Inset: scheme of one period of the object; it consists in two perpendicular rectangular apertures $(120 \mathrm{~nm}$ length and $50 \mathrm{~nm}$ width) which centers are shifted of $150 \mathrm{~nm}$ along both $x$ and $y$ directions

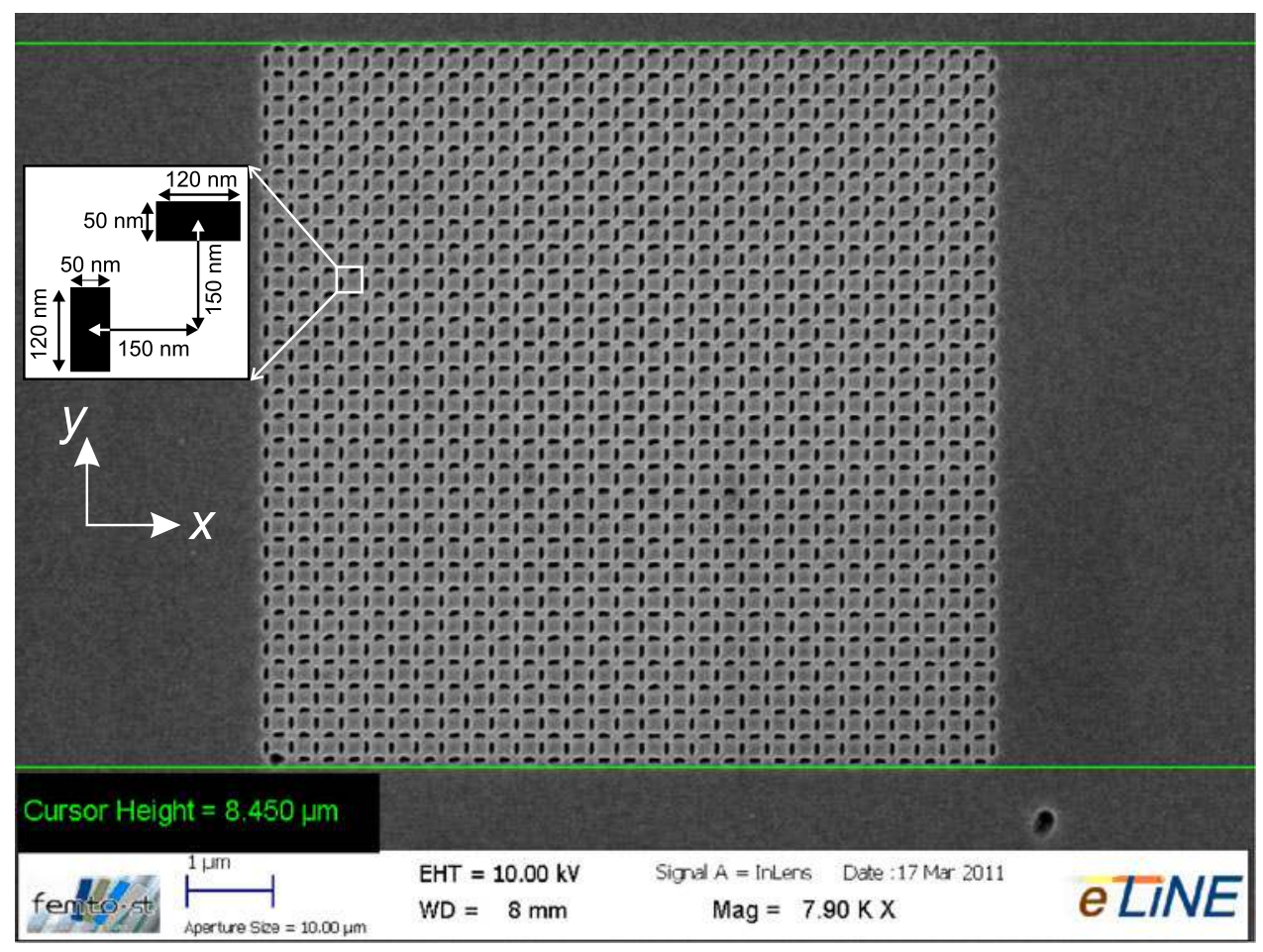

waveguide section behaves as if it is greater than the case of a perfect conductor [20]. Accordingly, the cutoff wavelength of the fundamental mode can be expressed by:

$\lambda_{c}^{\text {fund }}=2 a+\beta$,

where $\beta$ is a positive quantity which value depends on the metal dispersion through the skin depth inside the metal according to the guided mode electromagnetic field distribution. Thus, a numerical method (N-order FDTD algorithm) have been used to optimize the dimensions of the structure so that the cutoff wavelength is fixed to $\lambda=633 \mathrm{~nm}$ corresponding to the available continuous wavelength laser. We choose gold as a metal and its dielectric properties are described through a Drude-Lorentz model [21]. The length $a$ of the rectangle has been varied, keeping the width $b=50 \mathrm{~nm}$ corresponding to the smallest detail that can be reached by the used etching technique (see below). From numerical simulations, we deduced $a=120 \mathrm{~nm}$ for the rectangle length. This leads to $\beta \simeq 390 \mathrm{~nm}$ from (1) meaning an effective rectangle length of almost $315 \mathrm{~nm}$.

The next step consists in the experimental realization. For experimental constraints related to the difficulty of positioning the microscope probe above the structure, we choose to work with a periodic grating having an elementary pattern composed of two apertures. Indeed, the forthcoming results are valid for two isolated nano-apertures or for a periodic array of such nano-apertures. We must simply take the precaution of ensuring that no surface waves (mainly plasmons) can be excited in the case of an array of nano-structures: the Wood and Rayleigh anomalies must be kept away from the working wavelength. This condition can be easily fulfilled by imposing a small value of the period compared to $\lambda / n$ ( $n$ being the optical index of the substrate). This is why we fixed the grating period to $p=300 \mathrm{~nm}$ (the substrate is a glass microscope slide with $n \approx 1.5$ ).

The last step in the structure design is related to the relative position of the two apertures. To achieve a sub- $\lambda$ spatial control, the distance between the two aperture centers, where the electric field intensity confinement appears, should be smaller than $\lambda / 2$. To this end, we use a 3D-FDTD homemade code and we monitor the near-field optical response when varying this distance. Taking into account technological constraints and FDTD results, we have chosen the parameters indicated in the inset of Fig. 1.

\section{Experimental results}

A SEM image of the fabricated sample is shown in Fig. 1. The apertures have been fabricated by FIB milling in a $100 \mathrm{~nm}$ thick gold layer deposited on a glass substrate. The whole matrix is $8.45 \times 8.45 \mu \mathrm{m}^{2}$ size $(28 \times 28$ periods $)$. The SEM image shows very well-defined rectangular apertures and the periodicity is also well respected.

As explained before, the design of the fabricated structure (thickness, length, width, and nature of the metal) is optimized through theoretical calculations in order to get an enhanced transmission at a well-defined wavelength. In our case, this working frequency corresponds to the emission of 


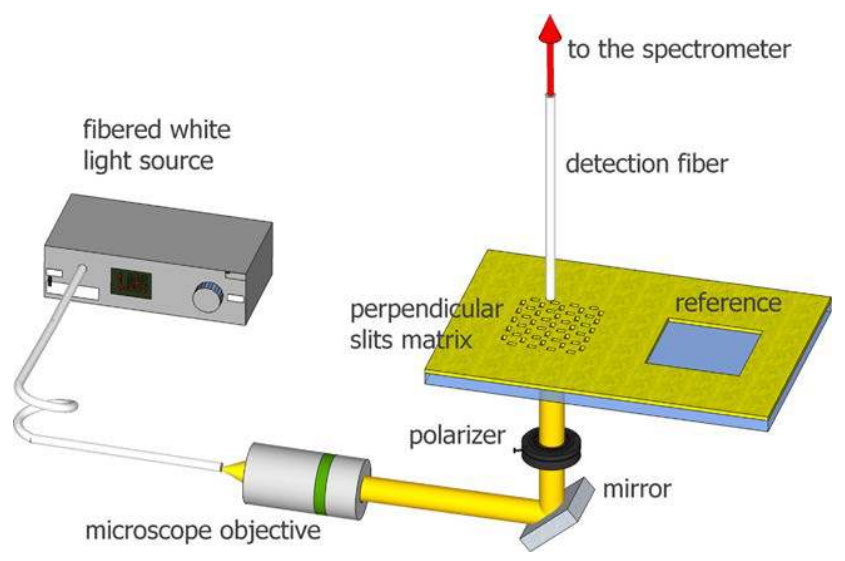

Fig. 2 Experimental setup used for the acquisition of the transmission spectra through the nano-structured metallic films

a red He-Ne laser $(\lambda=632.8 \mathrm{~nm})$. A Focused Ion Beam (FIB) milling combined with a very precise metal deposition process allows us to produce high quality rectangular apertures. A thin $5 \mathrm{~nm}$ titanium layer has been directly deposited onto a glass substrate to enhance the adhesion of the gold film (thickness $h=100 \mathrm{~nm}$ ) which is then deposited by evaporation. The FIB operates at $30 \mathrm{keV}$ and beam current is fixed to $12 \mathrm{pA}$. These parameters lead to an ion beam diameter of about 30-40 nm. SEM image in Fig. 1 shows the good quality of the fabricated apertures.

Two different experimental characterizations are performed in both far-field and near-field domains. The first one consists in recording the transmission spectrum while the second one addresses the light distribution in the nearfield through the use of a Scanning Near-field Optical microscope (SNOM) working in transmission mode. Consequently, two different experimental setups have been developed. The experimental transmission spectrum is recorded with the configuration presented in Fig. 2. A $200 \mathrm{~mW}$ white light source covering the spectral range [200 nm, $2400 \mathrm{~nm}$ ] is used. The incident beam illuminates the sample under normal incidence and a polarizer set a linear polarization along one of the axis of the grating. The transmitted light through the structure is collected by a cleaved end multi-mode optical fiber (core diameter of $62.5 \mu \mathrm{m}$ ). The fiber end is placed in front of the sample at a distance of around $50 \mu \mathrm{m}$. The other end of the fiber is connected to an optical spectrometer.

The transmission coefficients are theoretically and experimentally defined as the ratio of the transmitted intensity of the diffracted zero order through the structure to the same quantity measured through a reference area consisting in a squared aperture having the same lateral size as the array of nano-apertures and engraved in the same metallic film.

The transmission spectra depicted in Fig. 3 exhibit a quite good agreement between theory and experiment. The main peak due to the excitation of the $\mathrm{TE}_{01}$ mode reaches $45 \%$ in

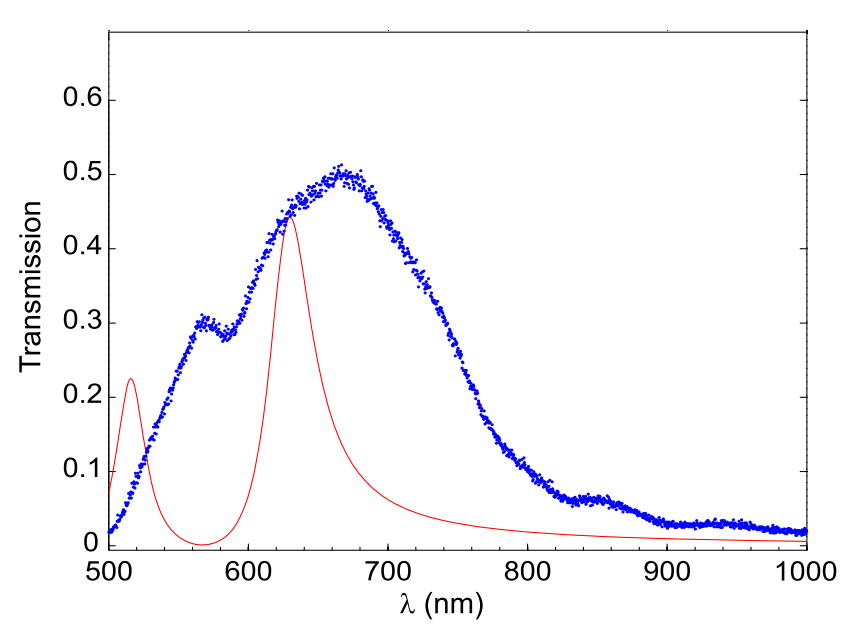

Fig. 3 Comparison between theoretical (solid red line) and experimental (dotted blue line) transmission spectra in the case of the structure of Fig. 1

both cases while the discrepancies between the two curves (half-width at half maximum, $50 \mathrm{~nm}$ red-shift of the peak) are mainly attributed to the nano-technological fabrication process of the apertures inducing slightly sloped vertical walls (V-shape). Nevertheless, the experimental transmission spectrum shows that the fabricated sample is suitable for near-field measurements at $\lambda=633 \mathrm{~nm}$. Note that the sub-peak theoretically located around $520 \mathrm{~nm}$ corresponds to the first harmonic of a Fabry-Perot resonance while the first peak at $\lambda=630 \mathrm{~nm}$ is associated to the harmonic zero [22].

SNOMs allow a resolution below the diffraction limit, thus they have been widely used to investigate the nearfield above nano-structured arrays and its role in extraordinary transmission [23-30]. In order to characterize the optical near-field above our sample, this latter is illuminated by a non-polarized parallel He-Ne laser beam under normal incidence (see Fig. 4). A polarizer allows to set the incident polarization along one of the grating axis ( $x$ or $y$ ). The transmitted optical near field is detected above the sample through a dielectric tip and both optical and shear-force images are recorded thanks to a commercial SNOM (NTMDT). The obtained images are shown in Fig. 5. The polarization is switched from the $x$ (bottom of the image) to the $y$ (top of the image) direction at the middle of the image (white dashed line) so that the vertical apertures are excited in the lower part of the image and the horizontal ones in its upper part (the fundamental $\mathrm{TE}_{01}$ mode is excited for a polarization parallel to the shortest side of the rectangular slit). The shear force image distinctly shows the grating of vertical and horizontal slits while the optical image clearly reveals vertical lines in the bottom of the image ( $x$-polarization) and horizontal lines in the top ( $y$-polarization). The period of these lines is about $300 \mathrm{~nm}$ which corresponds to the structure period. Moreover, one can distinguish in these lines hot spots 


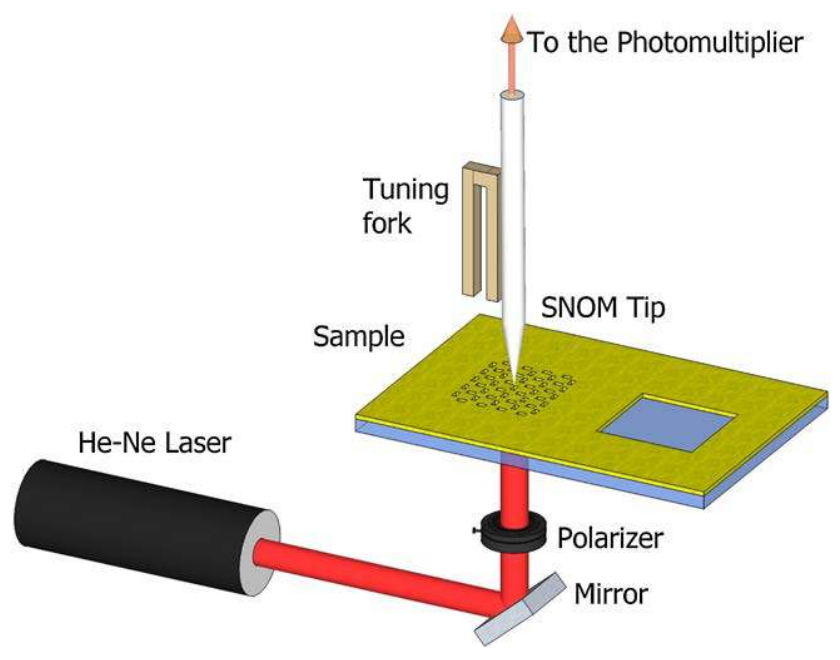

Fig. 4 Experimental setup used for the SNOM characterization. The tip-sample distance is kept constant during the scanning using an electronic feedback on the shear force signal given by the tuning fork

corresponding to the exact position of the slits, in agreement with the sample period of $300 \mathrm{~nm}$.

Figure 6 is a zoom-in on the optical image around the polarization switch area. The black-line rectangles mark the position of the slits that is deduced from the shear force image. One notes that the apertures are not exactly oriented along the $x$ - and $y$-directions. Actually, there is around 5 degrees deviation. This image proves that only the $\mathrm{TE}_{01}$ mode is excited in each aperture. Indeed, one can clearly see bright spots above the slits perpendicular to the polarization and dark spots above the parallel ones. The image contrast is inverted when the polarization is $90^{\circ}$ rotated. The nonperfect distribution of the light inside the slits may be related to the non-alignment of the polarization along one edge of the slits. Nevertheless, these experimental optical images clearly demonstrate the sub- $\lambda$ localization of the near field because the nearest slit centers are only separated by almost $215 \mathrm{~nm}$ and it is possible to switch "off" or "on" only one of these apertures by simply shifting of $90^{\circ}$ the incident polarization. The theoretical near-field intensity is depicted in Fig. 7: the near-field intensity is calculated $17 \mathrm{~nm}$ above the sample surface and it is defined as $|E|^{2}=\left|E_{x}\right|^{2}+\left|E_{y}\right|^{2}$ because a dielectric tip (as used in the experimental part of the paper) only detects the transversal components ( $E_{x}$ and $\left.E_{y}\right)$ of the electric field [31]. Moreover, in order to match as best as possible the experimental conditions, the polarization is set to 5 and 95 degrees with respect to the $x$-axis, respectively, in the bottom and the upper parts of the image. As expected, only the slits perpendicular to the polarization direction are excited and Fig. 7 shows a good agreement with the experimental results in Fig. 6. The spot light size above each excited aperture is larger in this last figure compared to the theoretical results because of the integration over the
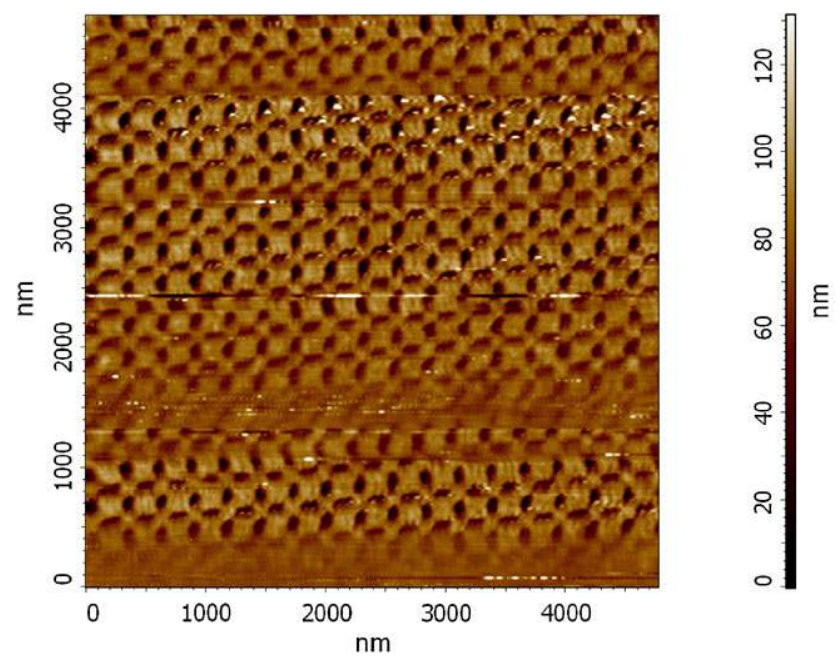

a Shear-force image.

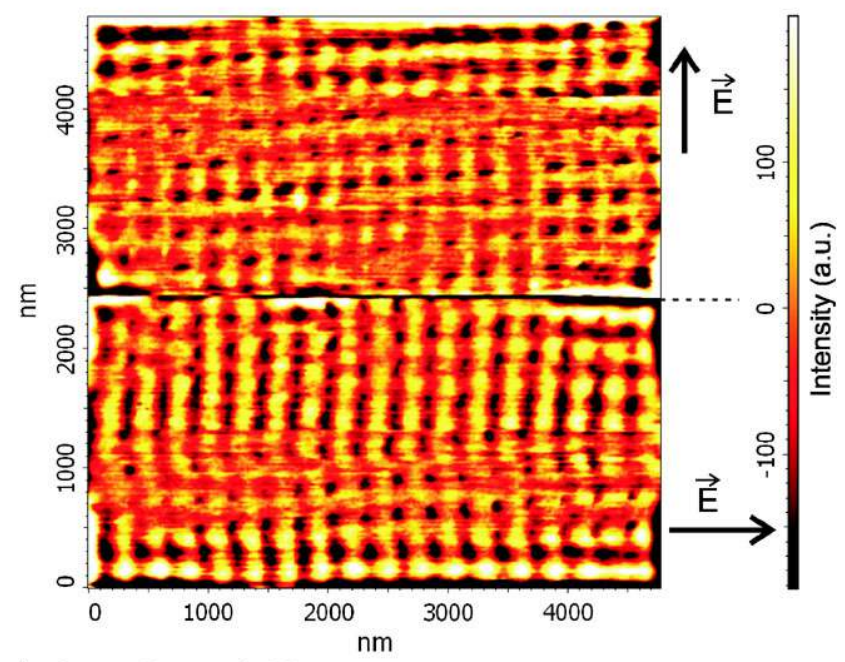

b Optical near-field image.

Fig. 5 (a) Shear force and (b) optical near-field images of the studied structure. The incident polarization is switched in the middle of the image: it is horizontal (i.e. along the $x$-axis) for the lower part and vertical (i.e. along the $y$-axis) for the upper part

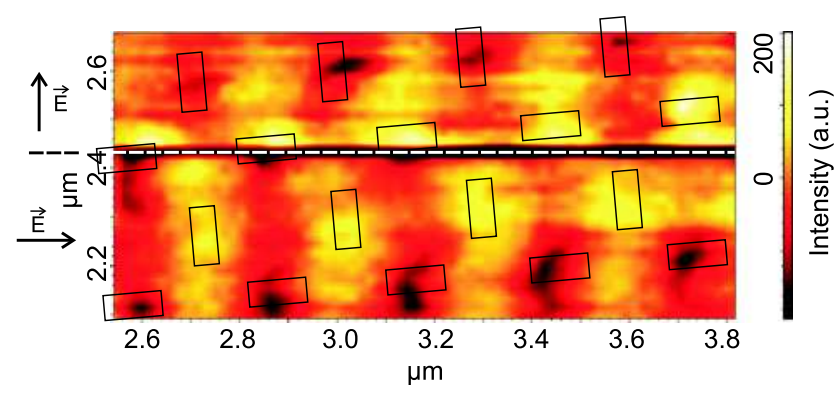

Fig. 6 Zoom-in over the near-field optical image (Fig. 5(b)) including the polarization switch (dashed line). The rectangles mark the position of the nano-slits deduced from the shear force image ( $5^{\circ}$ tilt). It is clear that the vertical slits are excited in the lower part of the image (horizontal polarization) and the horizontal ones in the upper part (vertical polarization) 
Fig. 7 Theoretical near-field intensity $|E|^{2}=\left|E_{x}\right|^{2}+\left|E_{y}\right|^{2}$ calculated (3D-FDTD) $17 \mathrm{~nm}$ above the gold film and corresponding to the experimental results of Fig. 6 . The dashed white rectangles mark the position of the non-excited apertures and the incident polarization is switched from horizontal direction to vertical one at the middle of the image (dashed white line). The polarization is set with a 5 degrees shift with respect to the axis in order to be consistent with the experimental conditions

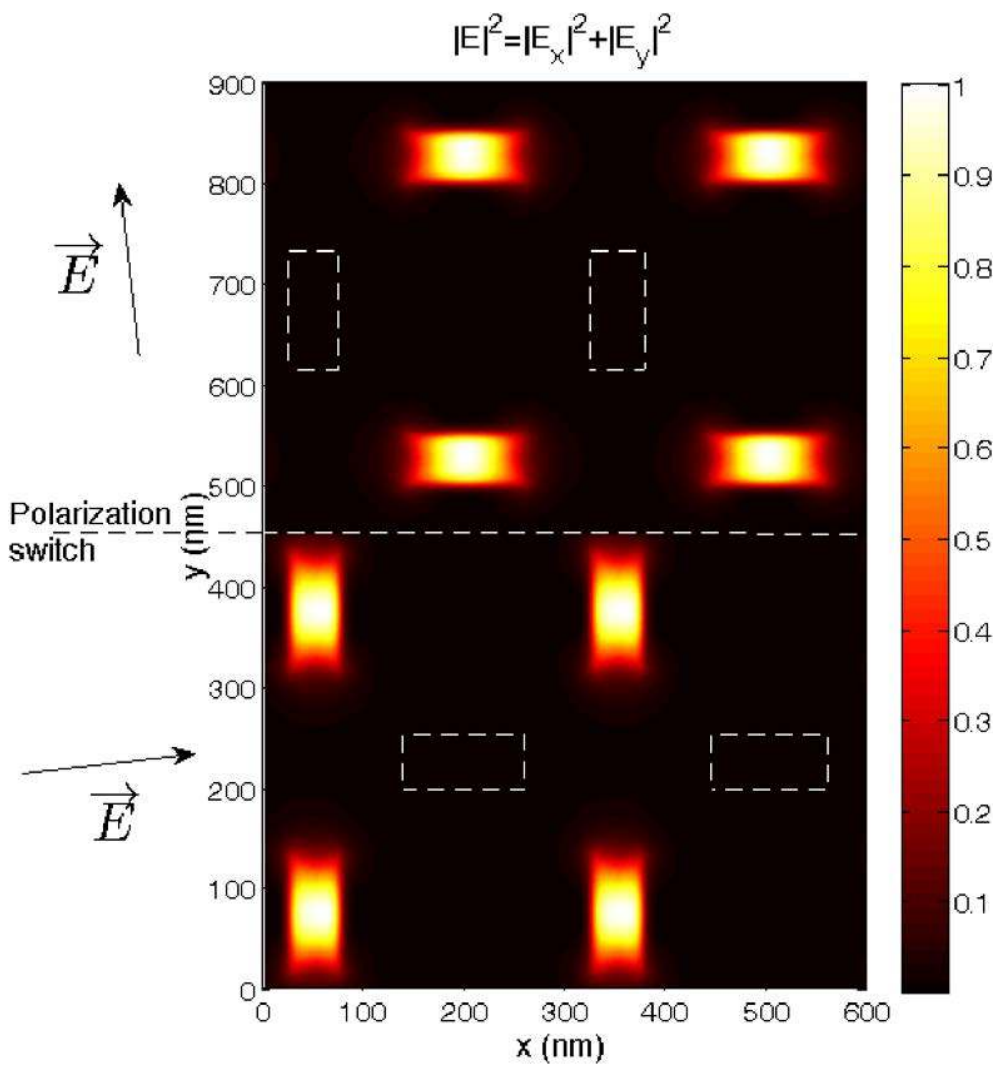

whole dielectric tip apex involved in the near field image process.

\section{Conclusion}

In summary, we have demonstrated a control of the near field light confinement in the vicinity of specific nano-structures by simply adjusting the linear polarization of the incident field. The experimental optical images clearly exhibit a subwavelength confinement because the distance between the centers of two nearest apertures is of only $215 \mathrm{~nm}$, i.e. $\lambda / 3$. Note that Caglayan et al. [6] showed an electromagnetic field localization into a region of $\lambda / 8$ thanks to split-ring resonators. However, they worked in the $\mathrm{GHz}$ regime while we work in the visible range meaning a large scale reducing. This kind of enhanced transmission nano-structures exploiting the excitation of a polarization sensitive guided mode inside the apertures offers a convenient and flexible way not only to confine but also to control light below the diffraction limit without the need of a material mediation such as near-field microscope probes.

Acknowledgements This work is partially supported by the Government of Senegal which supply A. Ndao's research grant and by the U.F.R. Sciences and Techniques of the University of FrancheComté which earmark funds. The authors would like to thank Roland Salut from the platform of technology, MIMENTO at FEMTO-ST, for greatly helping with the fabrication process.

\section{References}

1. C.C. Neacsu, S. Berweger, R.L. Olmon, L.V. Saraf, C. Ropers, M.B. Raschke, Nano Lett. 10, 592 (2010)

2. E.J. Sánchez, L. Novotny, X.S. Xie, Phys. Rev. Lett. 82, 4014 (1999)

3. F.I. Baida, Y. Poujet, B. Guizal, D. Van Labeke, Opt. Commun. 256, 190 (2005)

4. P. Ghenuche, S. Cherukulappurath, T.H. Taminiau, N.F. Van Hulst, Phys. Rev. Lett. 101, 116805 (2008)

5. G. Volpe, S. Cherukulappurath, R.J. Parramon, G. Molina-Terriza, Nano Lett. 9, 3608 (2009)

6. H. Caglayan, I. Bulu, M. Loncar, E. Ozbay, Opt. Lett. 34, 88 (2009)

7. E. Cubukcu, K. Aydin, E. Ozbay, S. Foteinopoulou, C.M. Soukoulis, Phys. Rev. Lett. 91, 207401 (2003)

8. Z. Liu, H. Lee, Y. Xiong, C. Sun, X. Zhang, Science 315, 1686 (2007)

9. J.B. Pendry, Phys. Rev. Lett. 85, 3966 (2000)

10. M. Aeschlimann, M. Bauer, D. Bayer, T. Brixner, F.J. Garcia de Abajo, W. Pfeiffer, M. Rohmer, C. Spindler, F. Steeb, Nature 446, 301 (2007)

11. S. Choi, D. Park, C. Lienau, M.S. Jeong, C.C. Byeon, D. Ko, D.S. Kim, Opt. Express 16, 12075 (2008)

12. G. Lévêque, O.J.F. Martin, Phys. Rev. Lett. 100, 117402 (2008)

13. X. Li, M.I. Stockman, Phys. Rev. B 77, 195109 (2008)

14. M.I. Stockman, D.J. Bergman, T. Kobayashi, Phys. Rev. B 69, $54202(2004)$

15. M.I. Stockman, S.V. Faleev, D.J. Bergmanl, Physica B 338, 361 (2003)

16. M. Sukharev, T. Seideman, Nano Lett. 6, 715 (2006)

17. W.L. Barnes, A. Dereux, T.W. Ebbesen, Nature 424, 824 (2003)

18. J.C. Weeber, Y. Lacroute, A. Dereux, E. Devaux, T.W. Ebbesen, T.C. Weeber, Phys. Rev. B 70, 235406 (2004) 
19. F.I. Baida, Opt. Express 18, 812 (2010)

20. R. Gordon, A.G. Brolo, Opt. Express 13, 1933 (2005)

21. F.I. Baida, D. Van Labeke, G. Granet, A. Moreau, A. Belkhir, Appl. Phys. B 79, 1 (2004)

22. F.I. Baida, D. Van Labeke, Opt. Commun. 209, 17 (2002)

23. M.H. Chowdhury, J.M. Catchmark, J.R. Lakowicza, Appl. Phys. Lett. 91, 103118 (2007)

24. D. Egorov, B.S. Dennis, G. Blumberg, M.I. Hafte, Phys. Rev. B 70, 033404 (2004)

25. H. Gao, J. Henzie, T.W. Odom, Nano Lett. 6, 2104 (2006)

26. S.C. Hohng, Y.C. Yoon, D.S. Kima, V. Malyarchukl, Appl. Phys. Lett. 81, 3239 (2002)
27. D.S. Kim, S.C. Hohng, V. Malyarchuk, Y.C. Yoon, Phys. Rev. Lett. 91, 143901 (2003)

28. E.S. Kwak, J. Henzie, S.H. Chang, S.K. Gray, G.C. Schatz, T.W. Odom, Nano Lett. 5, 1963 (2005)

29. M. Mrejen, A. Israel, H. Taha, M. Palchan, A. Lewis, Opt. Express 415, 9129 (2007)

30. Y. Poujet, M. Roussey, J. Salvi, F.I. Baida, D. Van Labeke, A. Perentes, C. Santschi, P. Hoffmann, Photonics Nanostruct. Fundam. Appl. 4, 47 (2006)

31. J. Greffet, R. Carminati, Prog. Surf. Sci. 56, 133 (1997) 